\title{
Unifying Fuzzy controller for Indoor Environment Quality
}

\author{
Miguel Molina-Solana, Maria Ros, Miguel Delgado \\ Department of Computer Science and Artificial Intelligence \\ Universidad de Granada, \\ Granada, Spain \\ Email: miguelmolina@ugr.es,marosiz@decsai.ugr.es, mdelgado@ugr.es
}

\begin{abstract}
Optimizing energy consumption while maintaining an appropriate level of comfort is one of the current challenges in the Indoor Environment Quality (IEQ) field. In this paper, we propose an unified fuzzy controller for managing the different aspects involved in IEQ, overcoming the potentially inefficient interactions between several traditional controllers. We also describe the implemented web simulator to test the controller, and the results of applying the controller to a pilot room, measuring the air quality over a period of a month. Even though, further research and experimentation should be done, specially with the experts in order to fine tuning the rules and the controller, initial results are promising enough and the ease of use is quite remarkable by users.
\end{abstract}

\section{INTRODUCTION}

Indoor Environment Quality (IEQ) has lately emerged as a topic of relevance once the presence of HVAC (heating, ventilation, and air conditioning) systems has been popularized in many buildings. IEQ tries to go beyond traditional control systems by introducing the comfort of the users in the game.

Reducing the energy consumption of those systems, while maintaining an appropriate comfort level is an issue of great interest that has not yet been fully addressed. Not in vain, energy consumption by the HVAC equipment in residential, commercial and industrial buildings represent about $40 \%$ of the total primary energy consumption (Intelligent Energy Executive Agency (IEEA), http://www.iea.org).

Traditionally, HVAC systems have not directly addressed user comfort, as they just tried to maintain the conditions within some limits. The optimization of that comfort is therefore completely dependent on how adapted is the system to user requirements. Conventional approach consists of On-Off and PID controllers (proportional-integral-derivative controllers), which try to minimize the error between the studied variable and the fixed setpoint throughout a defined mathematical model.

However, according to [1], HVAC systems could be considered as MIMO (Multiple Input Multiple Output) control problems, since they analyse interrelated variables to extract values for a set of outputs. Additionally, they are influenced by a great variety of uncertainty parameters, such as, external air temperature and occupants' activities or preferences, that change their normal operations. Therefore, HVAC control systems can be considered as multi-criteria problems described by means of complex analytical expressions.
Although traditional PIDs could provide reasonable solutions, they are not enough to control the uncertainty of the dynamics of HVAC systems, which are more easily characterised using linguistic labels and rules [2], [3]. Fuzzy Logic Controllers (FLCs) appear as a viable alternative to conventional controllers, since they do not required a mathematical modelling [4] and they are prepared to handle different criteria, as they represent the dynamic of the HVAC system according to the knowledge of a human expert. Moreover, their efficiency and lower energy consumption (while satisfying the indoor comfort requirements) comparing to PID controllers, has been completely demonstrated [5].

In the literature, an extensive variety of proposals in this direction can be found. For instance, Calvino et al. [6] focus on the development of a fuzzy controller for the control of the indoor thermal-hygrometry comfort conditions, allowing a better adjustment of the indoor micro-climate conditions.

The focus of the FLC is not only maintaining the thermal environment, but also optimizing other parameters if necessary. Becker et al. [7] propose a fuzzy controller for temperature and relative humidity $(\mathrm{RH})$ in refrigeration systems, by considering their thermodynamic coupling. In [8], authors develop a rulebased fuzzy controller to control and provide thermal comfort and adequate air distribution in a ventilated test room. Alcalá et al. [9] presented the use of genetic algorithms in order to develop optimized fuzzy logic controllers on the context of HVAC systems. As for Chen et al., they propose a FLC to control ventilation in road tunnels [10].

A step forward consists of applying fuzzy control strategies to the PID parameters in order to tune the HVAC control systems [11]. In [12], the authors make a correspondence between PID gains and FLC parameters, proving that for tracking room temperature, the designed Fuzzy-PID controller obtains better results than a traditional PID controller. As an additional advantage, this class of controller can be easily adapted to varying environments [13].

As an alternative to this interrelated design, we propose here a distributed control system with a Supervisory FLC that will reset and establish the set-points of the specific PID controllers with the aim of maintaining the Indoor Environment Quality (IEQ). Our FLC will take into consideration changes in both outdoor and indoor temperature, relative humidity levels and $\mathrm{CO}_{2}$ concentration, in addition to users' preferences, as the 
final objective. In order to test our proposal, we have developed a web simulator in which users manage FLC parameters with complete freedom, in order to obtain the most adjusted set of rules. Finally, the results of the user adjustment has been applied in a pilot room, where the environment quality over a period of a month has been measured and analysed to compare the performance of the PID controllers versus our Supervisory FLC.

This paper is organized as follows: in the next section, the designed Fuzzy Logic Controller is presented. In particular, details about its structure and the knowledge it incorporates are explained. In section III, we present the implemented web simulator to test the controller, whereas in section IV we show the results of the experimentation performed on the pilot room during a month. Finally, section V presents the conclusions and future works.

\section{Fuzzy Logic Controller Design}

Current HVAC systems are composed by a conventional controller (On-Off and PID controllers), changing the system operation according to a user-defined timetable. However, as already stated, HVAC systems are generally affected by a set of disturbances that could interfere with their correct operation; examples of those elements are external temperature, occupancy, building structure, etc.). Our proposal uses intelligent techniques to control both air quality and temperature of a building throughout improving the work of their individual HVAC systems.

Fuzzy control systems are most recommended in applications where the exact mathematical model for controlling them is not known, but their behaviour could be defined based on the experience. Using fuzzy control technology could achieve better flexibility by means of adjusting the system comfort requirements. Furthermore, expert rules, based on users knowledge, make the system more reliable to handle critical situations, and ease the scalability issues should more inputs are to be added to the controller.

The inputs to our system are five sensors (indoor and outdoor temperature, humidity, $\mathrm{CO}_{2}$ and lighting sensors), whereas the outputs are three actuators or outputs; in particular, air conditioner program, temperature setpoint and humidity level. Any decisions taken on one of the outputs could influence the general context, and, consequently, make changes in the others. A controller capable of taking into consideration those influences and reduce them as much as possible is required, and therefore, the use of a Fuzzy logic controller is completely justified.

The proposed fuzzy logic control scheme is presented in figure 1. Our proposal consists of attaching our Fuzzy Control Module to the current PID controllers with the aim of improving the flexibility and adjustment of the control system.

Therefore, the fuzzy logic controller's goal is to achieve user comfort within the monitored room. The operation of a FLC is based on the Inference Engine, which is responsible of accepting the inputs after the fuzzification process, and provide

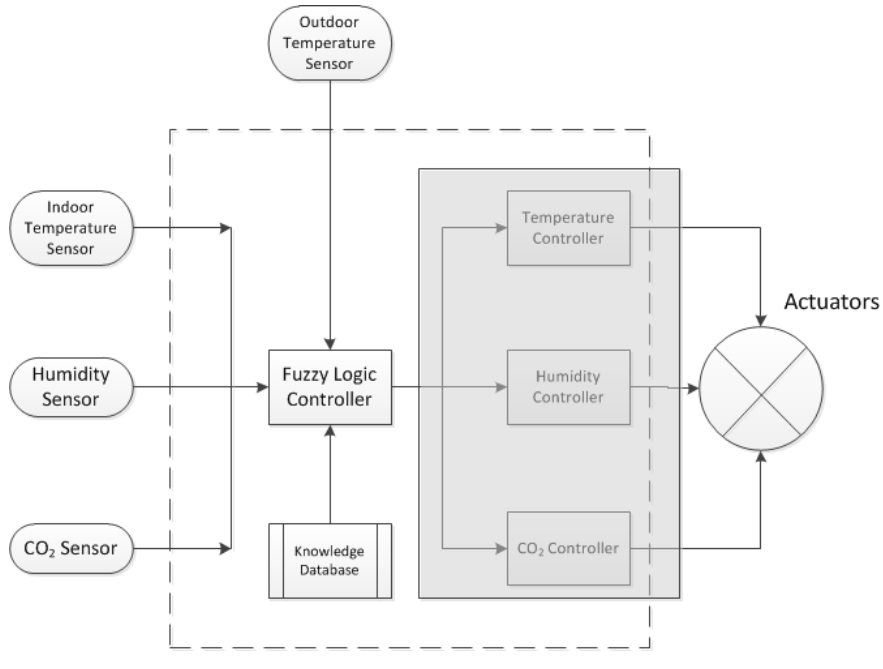

Fig. 1. Architecture PID and FLC controllers

the outputs values to the defuzzification module in order to obtain the output measurements.

The set of five distributed sensors (indoor and outdoor temperature, relative humidity and $\mathrm{CO}_{2}$ concentration sensors, lighting level) monitor the room continuously. From now on, we will named sensors $S_{\text {temp }}$ indoor,$S_{\text {temp }}$ outdoor,$S_{R H}$, $S_{\mathrm{CO}_{2}}$ and $S_{\text {light }}$, respectively. Those sensors measure the context of the monitored room, which will be used to infer the adequate behaviour of the system and, in particular, the instructions to apply on the actuators. We manage the air conditioner program, HVAC temperature setpoints, lighting level and humidifier functioning.

The Knowledge base collects the expert knowledge about the dynamic behaviour of the system. It is represented as a set of IF-THEN rules and membership functions. The current membership functions for the different linguistic tags have been defined by means of experts. In future works, we expect to learn those functions from real data.

The domain of every input is described over a set of three linguistic labels: Low, Medium and High. The particular membership functions depend on each input, but are always in the shape of Trapezoidal functions, defined by equation 1 . Figure 2 shows the different trapezoidal membership functions for the input sensor measurements.

$$
\mu_{L}\left(x_{i}\right)=\left\{\begin{array}{llc}
0 & \text { if } & x_{i}<a o x>d \\
\frac{x_{i}-a}{b-a} & \text { if } & a \leq x_{i} \leq b \\
1 & \text { if } & b \leq x_{i} \leq c \\
\frac{d-x_{i}}{d-c} & \text { if } & c \leq x_{i} \leq d
\end{array}\right.
$$

where $L$ can be Low, Medium and High, $a$ and $d$ are the end points of the trapezoidal membership function, $b$ and $c$ are the peak points of the trapezoidal membership function, and $x_{i}$ is the $i$ th sensor.

On the other hand, regarding the outputs' linguistic variables, they are defined depending on how the actuator works:

- Air conditioner program $\left(A_{A i r}\right)$ - hot, cold, dry 

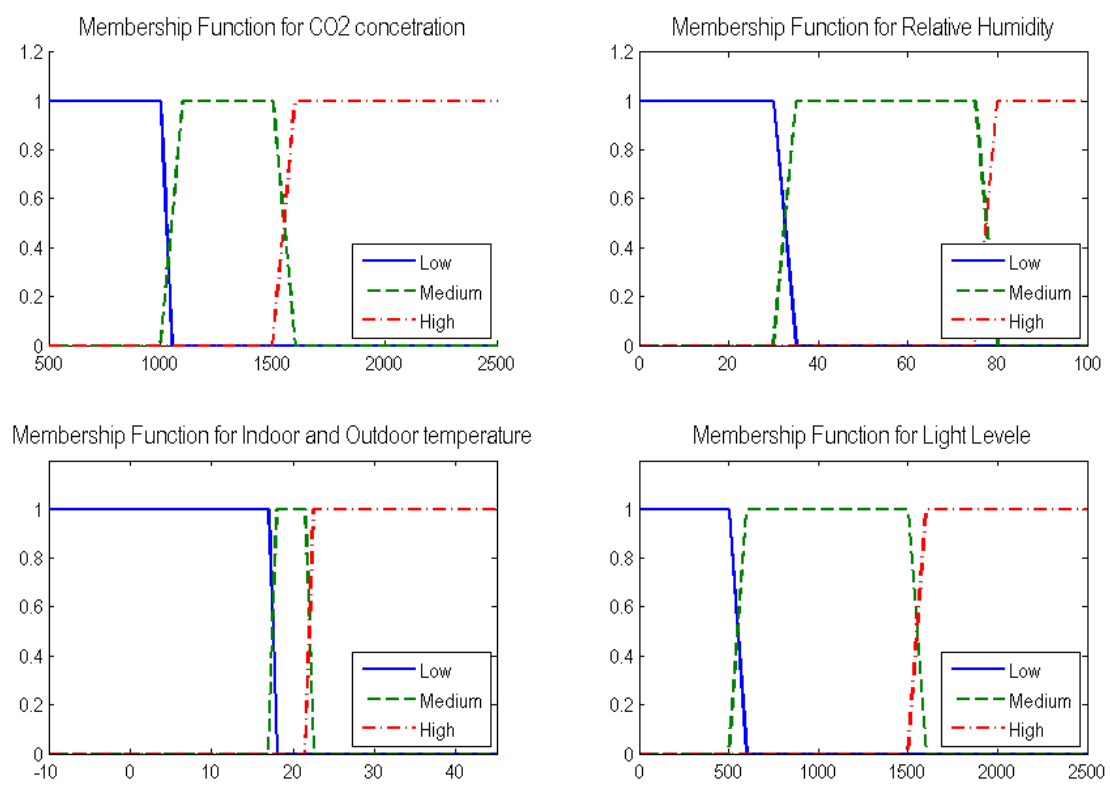

Fig. 2. Membership Function of the input variables

- Temperature setpoint $\left(A_{\text {temp }_{\text {level }}}\right)$ - down, hold, up

- Lighting level $\left(A_{\text {ligth }}\right)$ - low, medium, high

- Humidifier level $\left(A_{h_{\text {level }}}\right)$ - off, low, standard, high, continuous

Figure 3 shows a graphic representation of these linguistic variables.

Finally, we build the Fuzzy rules from expert knowledge and describe the quantitative relationship between variables in linguistic terms. Seventeen rules are used to determine the running of the FLC. They lead to different scenarios combining the possible inputs to the system. Table I shows a subset of the defined rules.

The inference engine applies Mamdani maxmin method [14] during the operation time:

$$
\begin{aligned}
& \mu_{R_{i}}(x)=\alpha_{i 1} \wedge \alpha_{i 2} \wedge \alpha_{i 3} \wedge \alpha_{i 4} \wedge \alpha_{i 5} \\
& \mu_{\text {Output }_{i}}(x)=\max _{R_{1}}(x), \mu_{R_{2}}(x), \ldots, \mu_{R_{17}}(x)
\end{aligned}
$$

where $\mathrm{x}$ is the input sensor measurements, $\alpha_{i}$ is the degree of a given input that satisfies the condition of the $i$ th rule $\left(R_{i}\right)$, and $\mu_{\text {output }_{i}}$ is the aggregation of fuzzy set outputs from all rules for output . $_{\text {. }}$

\section{Simulator SET-UP}

In order to test the devised fuzzy controller, we developed a simulator with a web interface, allowing us to define comfort parameters, give inputs and receive outputs from the controller. In particular, the simulator enables us to control temperature, lightning, humidity and $\mathrm{CO}_{2}$ concentration within a room by means of sensing all those variables and giving outputs in terms of, among others, modifying the HVAC temperature setpoint, activating lights or controlling the humidifier.
The simulator was designed in such a way that the outputs are given as actions to be taken, with its defuzzificated degree (if applicable).

The interface of the simulator is organized in three panels (Figures 4, 5 and 6) corresponding to the definition of the comfort parameters, the inputs, and the outputs of the simulator, respectively.

Figure 4 shows the panel of the interface in which the different intervals of comfort can be indicated. These parameters define the exact values of the different tags the controller use in its rules. The rules, as seen in Table I, has been in all cases previously given to the controller in fuzzy terms. The current version of the controller, and therefore the simulator, manages the indoor comfort in terms of lighting, $\mathrm{C} 02$ concentration, relative humidity and temperature. Other variables might very well be included with no additional complication.

The second panel (Figure 5) enables the introduction of inputs to the simulator. These inputs represent the state of the room in a particular timepoint. When tested outside of the simulator, these inputs would be connected to the corresponding sensors providing those measures. Logically, the variables for which the simulator expects inputs are those that appears in the antecedents of the controller's rules.

The last panel (Figure 6) shows the actions to be taken according to the controller outputs. Those actions' goals are transforming the current state of the room (given by the inputs) to an state in which all values are within the comfort intervals (as defined in the corresponding panel). The actions are expressed with a written instruction and with the degree to which it should be applied (when applicable). They are also illustrated with a picture. 

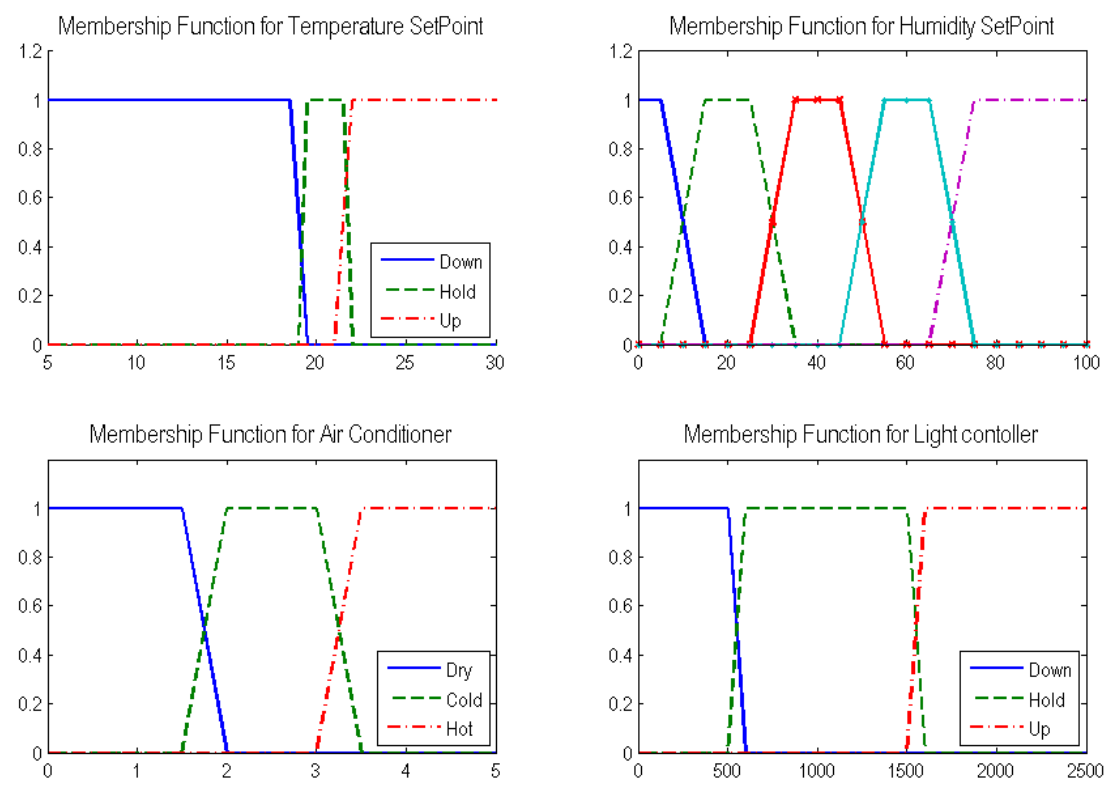

Fig. 3. Membership Function of the output variables

TABLE I

SAMPLE OF SET OF RULES

\begin{tabular}{l} 
IF $S_{\text {temp }}$ indoor IS Medium AND $S_{\text {tempoutdoor }}$ IS High THEN $A_{\text {temp }} p_{\text {level }}$ IS Hold AND $A_{h_{\text {level }}}$ IS Standard \\
IF $S_{R H}$ IS Low AND $S_{\text {temp }}$ indoor \\
IS Low THEN $A_{A i r}$ IS Hot AND $A_{h_{\text {level }}}$ IS High AND $A_{\text {temp }}$ IS Up \\
IF $S_{R H}$ IS Medium AND $S_{\text {temp }}$ IS High AND $S_{t e m p_{\text {indoor }}}$ IS Medium THEN $A_{A i r}$ IS Dry AND $A_{h_{\text {level }}}$ IS Continuous \\
\hline
\end{tabular}

Attending to Figures 4 to 6, we can check the following:

- When both the indoor and outdoor lighting are low, artificial lighting should be activated to solve the situation.

- Because the indoor relative humidity (19\%) is under the comfort threshold $(30 \%)$ the humidifier should be used.

- Because the indoor temperature is under the comfort temperature, and the outdoor temperature is also low, the window should be closed and the indoor temperature increased by means of the HVAC.

Those results are consistent with the expectations of the energy experts who identified the rules. In fact the simulator allowed an iterative process in which the experts keep refining the rules.

\section{EXPERIMENTAL SET-UP}

In addition to the aforementioned simulator, we have tested the fuzzy controller in a real environment: a room equipped with several sensors. Unfortunately, the room was not fullyequipped, lacking some of the sensors needed for IEQ control. Therefore we were forced to restrict ourselves to only control temperature and humidity.

The dimensions of the room were $5.2 \mathrm{~m} \times 5.2 \mathrm{~m} \times 2.5 \mathrm{~m}$. The room had a door in the North façade, and a window

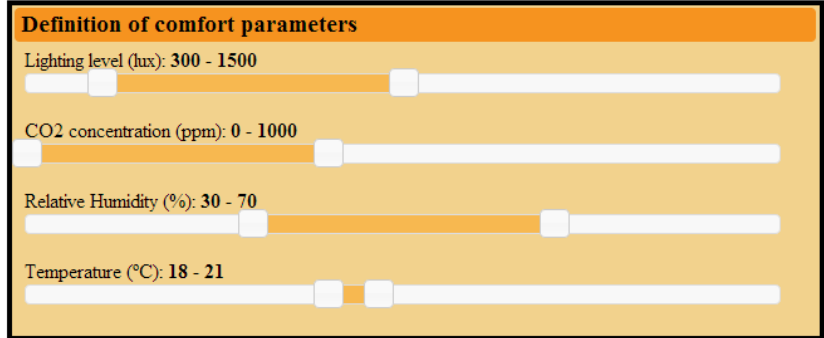

Fig. 4. Definition, in the simulator interface, of comfort parameters for the controller.

in the South one. During our experimentation, the room was completely empty (but for the sensors and the HVAC) with no furniture or people inside. However, the door was opened several times and occasionally some people entered for a few minutes. Although an empty room is not a realistic environment and thus not generally considered in the area, we wanted to model the room without external interferences. Further experiments will consider the presence of people and other objects.

Data were collected every 15 minutes during the last December and saved to a database. 


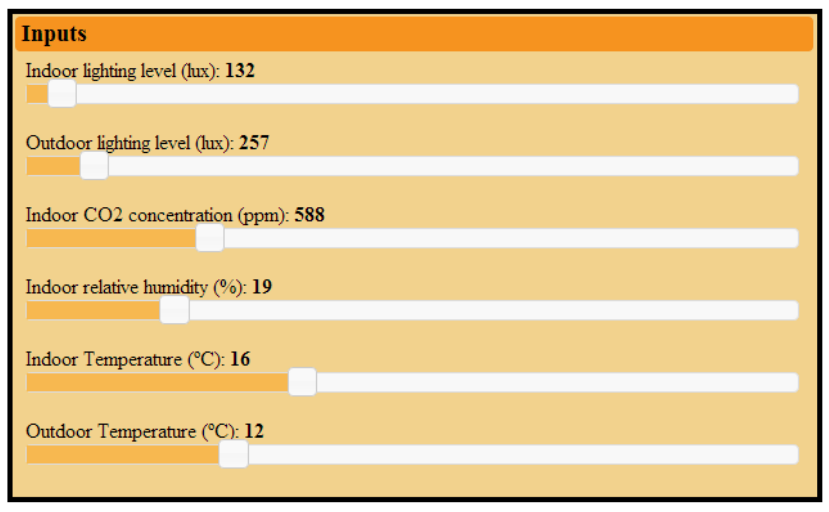

Fig. 5. Simulator panel for introducing the values of the different inputs.

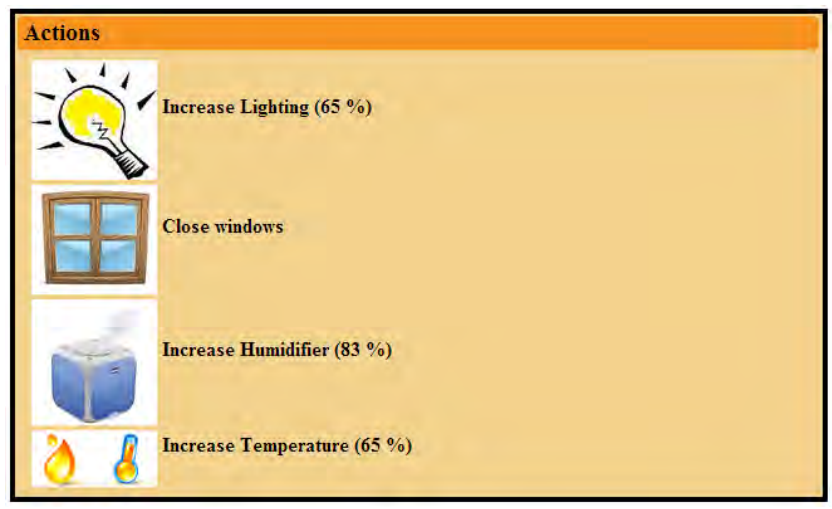

Fig. 6. Outputs of the simulator: actions to be taken.

As previously said, different fuzzy comfort rules were defined on the controller in order to manage the different actuators in the room, in particular the HVAC.

\section{A. Results}

In this section, we present the results obtained from running the developed fuzzy controller on the pilot room over a period of time of almost a month. We compare the obtained data with measures from a different period of time in which a reactive controller was running (instead of the fuzzy controller).

In Figure 7 data from indoor and outdoor temperatures are plotted. The data cover almost the whole month of December 2012. The gray area indicates the period in which the fuzzy controller was working. In the white area, simple reactive controllers were active. Those two distinct periods allow us to compare the performance of the different controllers. From Figure 7 it is very remarkable how the fuzzy controller is able to keep the temperature in the comfort zone, and also maintain it very stable. The reactive controller is also able to maintain the indoor temperature within the limits, but with a very appreciable oscillating movement.

On the other hand, Figure 8 shows the indoor and outdoor relative humidity. From the plot, although not so clear as in the temperature one, we can see how the fuzzy controller (gray area) do a great job in maintaining humidity within the comfort zone. Again, the oscillations are much more stable when using the fuzzy controller compare to the first part of the month, in which the reactive controller was running.

\section{Conclusion}

We have described in this paper an integrating fuzzy controller to manage different variables affecting the Indoor Environment Quality in a room. This fuzzy controller allows us to unify different controllers into only one that controls several variables being fully aware of the interdependencies between variables.

In addition to designing and implementing the fuzzy controller, we have implemented a simulator that allows us to indicate the different comfort intervals and inputs, and to test the feasibility of the fuzzy controller.

We have also applied the fuzzy controller to a real room in order to control the temperature and humidity within the room. Results were very promising and we have shown how temperature within the room keeps very stable when the fuzzy controller was running, contrasting with those periods of time in which a pure reactive controller was running. Regarding humidity, the fuzzy controller is again able to maintain the humidity in the room within the comfort interval, diminishing and letting it increase when neccesary.

What is relevant in our approach is that we are addressing several controls by means of a unique controller that is fully aware of all the sensors and actuators and the potential interactions among them that, in many cases, could lead to contradictory decisions and inefficiencies.

Despite only preliminary testing have been carried out, results are very promising and encourage us to continue adjusting the fuzzy controller and applying it to more tasks. In this regard, we plan to install additional sensors and actuators in the pilot room in order to measure and control other variables. Among those, we have already tackled lighting and $\mathrm{CO}_{2}$ concentration in the simulator.

\section{ACKNOWLEDGMENTS}

The authors would like to thank the Spanish Ministry of Education for their funding under the project TIN2009-14538C02-01. Miguel Molina-Solana and María Ros are also funded by the Research Office at the University of Granada.

\section{REFERENCES}

[1] H. Mirinejad, K. Welch, and L. Spicer, "A review of intelligent control techniques in hvac systems," in Energytech, 2012 IEEE, 2012, pp. 1-5.

[2] T. Takagi and M. Sugeno, "Fuzzy identification of systems and its applications to modeling and control," Systems, Man and Cybernetics, IEEE Transactions on, vol. SMC-15, no. 1, pp. 116-132, 1985.

[3] L. Zadeh, "Fuzzy sets and systems," Int J General Syst, vol. 17, pp. 129-138, 1990.

[4] S. Soyguder, M. Karakose, and H. Alli, "Design and simulation of selftuning pid-type fuzzy adaptive control for an expert hvac system," Expert Systems with Applications, vol. 36, no. 3, Part 1, pp. 4566-4573, 2009.

[5] D. Kolokotsa, "Comparison of the performance of fuzzy controllers for the management of the indoor environment," Building and Environment, vol. 38, no. 12, pp. 1439-1450, 2003.

[6] F. Calvino, M. L. Gennusa, G. Rizzo, and G. Scaccianoce, "The control of indoor thermal comfort conditions: introducing a fuzzy adaptive controller," Energy and Buildings, vol. 36, no. 2, pp. 97-102, 2004. 


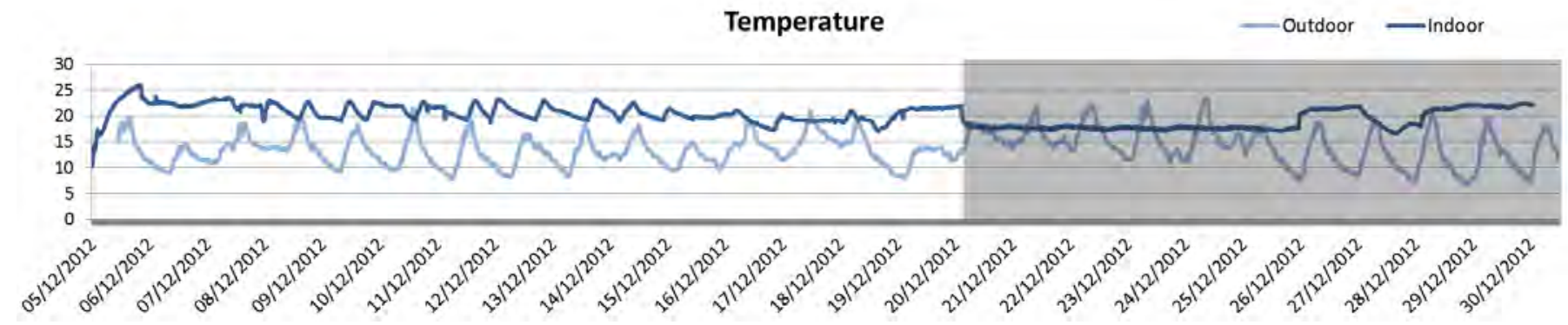

Fig. 7. Indoor and outdoor temperatures gathered in the room last December. The gray areas are those in which the fuzzy controller was running.

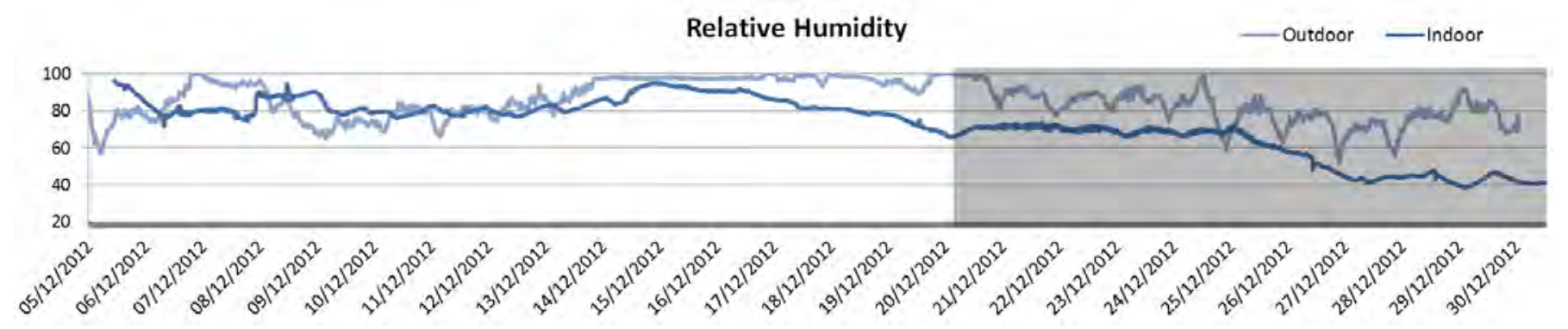

Fig. 8. Indoor and outdoor humidity data gathered in the room last December. The gray areas are those in which the fuzzy controller was running.

[7] M. Becker, D. Oestreich, H. Hasse, and L. Litz, "Fuzzy control for temperature and humidity in refrigeration systems," in Control Applications, 1994., Proceedings of the Third IEEE Conference on, vol. 3, 1994, pp. $1607-1612$.

[8] M. Eftekhari and L. Marjanovic, "Application of fuzzy control in naturally ventilated buildings for summer conditions," Energy and Buildings, vol. 35, no. 7, pp. 645-655, 2003.

[9] R. Alcal, J. Bentez, J. Casillas, O. Cordn, and R. Prez, "Fuzzy control of hvac systems optimized by genetic algorithms," Applied Intelligence, vol. 18 , no. 2, pp. 155-177, 2003.

[10] P.-H. Chen, J.-H. Lai, and C.-T. Lin, "Application of fuzzy control to a road tunnel ventilation system," Fuzzy Sets and Systems, vol. 100, no. 1-3, pp. 9-28, 1998.

[11] Z.-Y. Zhao, M. Tomizuka, and S. Isaka, "Fuzzy gain scheduling of pid controllers," Systems, Man and Cybernetics, IEEE Transactions on, vol. 23, no. 5, pp. 1392-1398, 1993.

[12] L. Hongli, D. Peiyong, and J. Lei, "A novel fuzzy controller design based-on pid gains for hvac systems," in Intelligent Control and Automation, 2008. WCICA 2008. 7th World Congress on, 2008, pp. 736-739.

[13] A. Dounis and C. Caraiscos, "Advanced control systems engineering for energy and comfort management in a building environment-a review," Renewable and Sustainable Energy Reviews, vol. 13, no. 6-7, pp. 1246$1261,2009$.

[14] E. Mamdani, "Application of fuzzy logic to approximate reasoning using linguistic synthesis," Computers, IEEE Transactions on, vol. C-26, no. 12, pp. 1182-1191, 1977. 